\title{
Elevated blood viscosity during exercise: What are the consequences?
}

\author{
Philippe Connes \\ Laboratoire ACTES, Département de Physiologie, Université des Antilles et de la Guyane, Campus de \\ Fouillole, Pointe-à-Pitre, Guadeloupe and UMR S 763 Inserm/Université des Antilles et de la Guyane, \\ CHU Pointe-à-Pitre, Pointe-à-Pitre, Guadeloupe \\ E-mail: pconnes@yahoo.fr
}

I read with interest the recent Editorial published by Forconi et al. [6]. Hyperviscosity is classically admitted to disturb blood flow and to represent a risk factor for the development of cardiovascular disease. However, recent experimental results tackle this view and support the hypothesis that hyperviscosity might represent a potential compensatory mechanism in the regulation of peripheral resistances and tissue perfusion in the setting of disease [6]. The resulting increased wall shear stress may stimulate nitric oxide production by endothelial cells to create a vasodilatory compensation [8].

Exercise induces important hematological/hemorheological changes such as an increase of hematocrit and blood viscosity [1]. Connes et al. [3] observed that athletes who developed exercise induced hypoxemia (EIH; i.e. with a significant decrease of arterial pressure in oxygen of more than $4 \%$ as compared to resting value) exhibited a greater increase of blood viscosity during exercise than those without EIH. It was hypothesized that the higher blood viscosity reached might increase the pulmonary artery pressure and damage the alveolar-capillary membrane through the stress-failure mechanism resulting in pulmonary oxygen diffusion impairment [2]. However, recent data demonstrated that muscle oxygenation is greater in EIH athletes than in athletes without EIH during exercise and that is the greater muscle oxygenation that could explain the decrease of arterial pressure in oxygen [7]. The mechanisms at the origin of this greater muscle oxygenation are unknown. We recently studied hemodynamical, respiratory and hemorheological changes during exercise, independently of EIH, and we found that the increase of blood viscosity between rest and exercise was positively correlated with the enlargement of the arteriovenous oxygen content difference suggesting that the greater the increase of blood viscosity, the greater muscle oxygen consumption [5]. From a classical view point it could seem surprising but, in fact, the increase of blood viscosity, in association with the increase of blood flow, may increase shear stress and stimulate vasodilation through a NO-dependent mechanism and allow adequate oxygen delivery to tissues. The same mechanism may be advanced in athletes prone to EIH. The higher blood viscosity, and the resulting higher shear stress, during exercise might stimulate the endothelial production of nitric oxide and increase the functional capillary density at the muscle level leading to better tissue oxygenation.

Another paradox concerns the case of sickle cell trait (SCT) carriers. Several studies reported elevated resting and exercising blood viscosity (see [4] for review) and it is usually advanced that it could reduce the aerobic physical fitness in this population. However, aerobic physical fitness of SCT carriers 
is absolutely normal. One may hypothesize that the hyperviscosity of SCT carriers could, again, stimulate vasodilation and compensate for the negative effects of the other alterations (reduced red blood cell deformability, leucocyte/platelet activation) on blood flow and tissue oxygenation.

Clearly these hypotheses need to be verified but recent data on the beneficial effects of hyperviscosity on vascular function open a new promising and exciting way in the field of hemorheology and physiology.

\section{References}

[1] J.F. Brun, S. Khaled, E. Raynaud, D. Bouix, J.P. Micallef and A. Orsetti, The triphasic effects of exercise on blood rheology: Which relevance to physiology and pathophysiology?, Clin. Hemorheol. Microcirc. 19 (1998), 89-104.

[2] C. Caillaud, P. Connes, D. Bouix and J. Mercier, Does haemorheology explain the paradox of hypoxemia during exercise in elite athletes or thoroughbred horses?, Clin. Hemorheol. Microcirc. 26 (2002), 175-181.

[3] P. Connes, D. Bouix, F. Durand, P. Kippelen, J. Mercier, C. Prefaut, J.F. Brun and C. Caillaud, Is hemoglobin desaturation related to blood viscosity in athletes during exercise?, Int. J. Sports Med. 25 (2004), 569-574.

[4] P. Connes, H. Reid, M.D. Hardy-Dessources, E. Morrison and O. Hue, Physiological responses of sickle cell trait carriers during exercise, Sports Med. 38 (2008), 931-946.

[5] P. Connes, J. Tripette, M. Mukisi-Mukasa, O.K. Baskurt, K. Toth, H.J. Meiselman, O. Hue and S. Antoine-Jonville, Relationships between hemodynamic, hemorheologic and metabolic responses during exercise, Biorheology 46(2) (2009), $133-143$.

[6] S. Forconi and T. Gori, The evolution of the meaning of blood hyperviscosity in cardiovascular physiopathology: Should we reinterpret Poiseuille?, Clin. Hemorheol. Microcirc. 42 (2009), 1-6.

[7] R. Legrand, S. Ahmaidi, W. Moalla, D. Chocquet, A. Marles, F. Prieur and P. Mucci, O2 arterial desaturation in endurance athletes increases muscle deoxygenation, Med. Sci. Sports Exerc. 37 (2005), 782-788.

[8] B.Y. Salazar Vazquez, M.A. Salazar Vazquez, V.C. Venzor, A.C. Negrete, P. Cabrales, J.S. Diaz and M. Intaglietta, Increased hematocrit and reduced blood pressure following control of glycemia in diabetes, Clin. Hemorheol. Microcirc. 38 (2008), $57-64$. 